\title{
Publisher's Note: Space-time characteristics of wall-pressure and wall shear-stress fluctuations in wall-modeled large eddy simulation [Phys. Rev. Fluids 1, 024404 (2016)]
}

George Ilhwan Park and Parviz Moin (Received 12 April 2017; published 19 April 2017)

DOI: 10.1103/PhysRevFluids.2.049901

This paper was published online on 20 June 2016 with a typographical error in Table I. In Table I, the last row of the first column should read as "Incompressible DNS [26]." The table has been corrected as of 10 April 2017. 